Journal of Science
http:/dergipark.gov.tr/gujs

\title{
New Efficient Numerical Model for Solving Second, Third and Fourth Order Ordinary Differential Equations Directly
}

\author{
Olusola Ezekiel ABOLARIN ${ }^{1}$ (D) , John Olusola KUBOYE ${ }^{1}$ (D) , Emmanuel Oluseye ADEYEFA ${ }^{1}$ (D), Bamikole \\ Gbenga OGUNWARE2** \\ ${ }^{1}$ Mathematics Department, Federal University, Oye-Ekiti, Ekiti State, Nigeria \\ ${ }^{2}$ Mathematics and Statistics Department, Joseph Ayo Babalola University, Ikeji-Arakeji, Osun State, Nigeria
}

\section{Highlights}

- The paper focused on the numerical solution of higher order initial value problems.

- Power series was used as the basis function for the derivation of the method.

- The method solved second, third and fourth order ordinary differential equations concurrently.

- This method satisfied the basic properties of a linear multistep method.

- This method generated more accurate results than the existing numerical methods.

\section{Article Info}

Received: $01 / 10 / 2019$

Accepted: 01/06/2020

\section{Keywords}

Hybrid Block method

Collocation

Interpolation

Power series

Higher order

\begin{abstract}
This article presents a two-step hybrid linear multistep block method for solving second, third and fourth order initial value problems of ordinary differential equations directly. The derivation of the method was done using collocation and interpolation techniques, while approximated power series was used as an interpolating polynomial. The fourth derivative of the power series was collocated at the entire grid and off-grid points, while the fifth and sixth derivatives of the polynomial were collocated at the endpoint only. The basic properties of the developed method, that is, order, error constant, zero stability, region of absolute stability, convergence and consistency of the method were properly investigated. The numerical results demonstrated that the scheme developed handles: second, third and fourth order ordinary differential equations efficiently and accurately when compared with existing methods. The proposed method takes away the burden of developing a separate method for the solution of second, third and fourth order initial value problem of ordinary differential equations.
\end{abstract}

\section{INTRODUCTION}

A numerical method is an approach where difficult problems in mathematics are being solved. This technique provides an approximate solution to differential equations which are ordinary differential equations (ODEs) and partial differential equations (PDEs). Different researchers have worked on the development of numerical methods for solving these differential equations and this includes [1-4].

The direct numerical solution of second, third and fourth order initial value problem of ODEs of the system: $y^{\prime \prime}(x)=f\left(x, y, y^{\prime}\right), y\left(x_{0}\right)=y_{0}, y^{\prime}\left(x_{0}\right)=y_{1}$

$y^{\prime \prime \prime}(x)=f\left(x, y, y^{\prime}, y^{\prime \prime}\right), y\left(x_{0}\right)=y_{0}, y^{\prime}\left(x_{0}\right)=y_{1}, y^{\prime \prime}\left(x_{0}\right)=y_{2}$

$y^{\prime v}(x)=f\left(x, y, y^{\prime}, y^{\prime \prime}, y^{\prime \prime \prime}\right), y\left(x_{0}\right)=y_{0}, y^{\prime}\left(x_{0}\right)=y_{1}, y^{\prime \prime}\left(x_{0}\right)=y_{2}, y^{\prime \prime \prime}\left(x_{0}\right)=y_{3}$

is considered using a single linear multistep hybrid technique in this research. 
There are two approaches to solving Equation (1). The first approach is to reduce Equation (1) above to its equal system of first order ordinary differential equations, and to solve using the appropriate method as highlighted by [5-7]. The second approach for solving (1) is using a direct method as proposed by [8-11]. These methods give the numerical solution one point at a time, but the results are poor in terms of accuracy.

To overcome these challenges and bring improvement on the numerical method, [12-14] developed block procedures for solving higher order ODEs directly. Here, the correctness of the procedures is better than when it is reduced to the arrangement of first order ODEs. Similarly, [14-16] established a hybrid block method for the direct solution of the general second, third and fourth order initial value problem of ODEs respectively. Generalized hybrid technique for solving second order ordinary differential equations directly was also done by [17]. [18] presented a direct two-point parallel block method for solving third and fourth order ODEs. [19] built a 2-point block mode for solving first and second order ODEs using different step size.

In this study, the usage of a lone hybrid block linear multistep method for the solution of second, third and fourth order ordinary differential equations which are uncommon in Numerical Analysis literature will be our focus.

Therefore, we are going to extend the work done in [18] and [19] by implementing a 2-step hybrid technique in block mode to solve second, third and fourth order ordinary differential equations directly.

\section{RESEARCH METHODOLOGY}

\subsection{The Development of the New Numerical Technique}

We considered power series as an estimated solution to Equation (1) to be the model

$$
y(x)=\sum_{j=0}^{k+9} a_{j} x^{j}
$$

where $a_{j}{ }^{\prime} s$ are parameters to be determined and $\mathrm{k}$ is the step-length.

The fourth, fifth and sixth derivatives of (2) are obtained as:

$$
\begin{aligned}
& y^{i v}(x)=\sum_{j=0}^{k+9} j(j-1)(j-2)(j-3) a_{j} x^{j-4} \\
& y^{v}(x)=\sum_{j=0}^{k+9} j(j-1)(j-2)(j-3)(j-4) a_{j} x^{j-5} \\
& y^{v i}(x)=\sum_{j=0}^{k+9} j(j-1)(j-2)(j-3)(j-4)(j-5) a_{j} x^{j-6} .
\end{aligned}
$$

Collocating the fourth derivative at all the grid and off-grid points $x=x_{n+j}$, where $j=0, \frac{1}{2}, 1, \frac{3}{2}, 2$ and the fifth and sixth derivatives are collocated at $x=x_{n+j}$, where $j=2$. The base function is being interpolated at $x=x_{n+j}, j=0, \frac{1}{2}, 1, \frac{3}{2}$. These equations were then combined to generate a structure of nonlinear system of equations which were solved using Gaussian Elimination Method. The resulting values generated were substituted back to the power series to give a continuous hybrid formula of the form in Equation (6)

$$
y(x)=\sum_{j=0}^{k-1} a_{j}(x) y_{n+j}+h^{4}\left(\sum_{j=0}^{k} \beta_{j}(x) f_{n+j}+\beta_{v}(x) f_{n+v}\right)+h^{5}\left(\sum_{j=0}^{k} \gamma_{j}(x) g_{n+j}\right)+h^{6}\left(\sum_{j=0}^{k} \tau_{j}(x) m_{n+j}\right)
$$


where $y(x)$ is the numerical solution of the initial value problem, $v=\frac{1}{2}, \frac{3}{2}$ are the hybrid points and $\alpha_{j}, \beta_{j}, \gamma_{j}$ and $\tau_{j}$ are constants. $f_{n+j}, g_{n+j}$ and $m_{n+j}$ are expressed in Equations (7), (8) and (9) as follows:

$f_{n+j}=y\left(x_{n+j}, y_{n+j}, y_{n+j}^{\prime}, y_{n+j}^{\prime \prime}\right) \quad j=0(1) k$

$g_{n+j}=y\left(x_{n+j}, y_{n+j}, y_{n+j}^{\prime}, y_{n+j}^{\prime \prime}, y_{n+j}^{\prime \prime \prime}\right) \quad j=0(1) k$

$m_{n+j}=y\left(x_{n+j}, y_{n+j}, y_{n+j}^{\prime}, y_{n+j}^{\prime \prime}, y_{n+j}^{\prime \prime \prime}, y_{n+j}^{\prime v}\right) \quad j=0(1) k$.

It should be noted that $\alpha_{0}, \beta_{0}, \gamma_{0}$ and $\tau_{0}$ are not zero since (3), (4) and (5) are contuous and differentiable; hence it is evaluated along with its derivatives at all the grid and off-grid points. This will produce a block method for general higher order ordinary differential equation of the type in Equation (10) below:

$A^{(0)} Y_{m}=A^{(i)} Y_{m-1}+h^{\mu}\left[B^{(i)} F_{m}+B^{(0)} Y_{m-1}\right]$

where

$Y_{m}=\left[y_{n+1}, y_{n+2}, \ldots, y_{n+r}\right]^{T}, Y_{m-1}=\left[y_{n-1}, y_{n-2}, \ldots, y_{n}\right]^{T}$,

$F_{m}=\left[f_{n}, f_{n+1}, f_{n+2}, \ldots, f_{n+k}\right]^{T}, F_{m-1}=\left[f_{n-1}, f_{n-2}, f_{n-3}, \ldots, f_{n}\right]^{T}, \mu$

is the order of the differential equation.

This gives the independent solution $\left\{y_{n+j}\right\}, i=1(1) k$ without overlapping.

Using the transformation,

$t=\frac{\left(x-x_{n+1}\right)}{h}, d t=\frac{1}{h} d x$

the coefficients of $y_{n+j}, g_{n+j}$ and $m_{n+j}$ are gotten in terms of t as follows:

$$
\begin{aligned}
& \alpha_{0}(t)=\left(-\frac{11}{3} t+4 t^{2}-\frac{4}{3} t^{3}+1\right) \\
& \alpha_{\frac{1}{2}}(t)=\left(4 t^{3}-10 t^{2}+6 t\right) \\
& \alpha_{1}(t)=\left(-4 t^{3}+8 t^{2}-3 t\right) \\
& \alpha_{\frac{3}{2}}(t)=\left(\frac{2}{3} t-2 t^{2}+\frac{4}{3} t^{3}\right) \\
& \beta_{0}(t)=h^{4}\left(\frac{131}{40320} t^{8}-\frac{31}{720} t^{5}-\frac{1}{2016} t^{9}+\frac{1}{24} t^{4}-\frac{7}{576} t^{7}+\frac{1}{30240} t^{10}-\frac{1397}{1290240} t-\frac{15893}{645120} t^{3}+\frac{41}{1440} t^{6}+\frac{30973}{3870720} t^{2}\right) \\
& \beta_{1}(t)=h^{4}\left(-\frac{73}{420} t^{7}-\frac{1}{5} t^{5}-\frac{2}{189}+\frac{33}{560} t^{8}-\frac{103}{17920} t^{2}+\frac{1}{1260} t^{10}-\frac{299}{53760} t+\frac{11}{189} t^{3}+\frac{5}{18} t^{6}\right) \\
& \beta_{\frac{3}{2}}(t)=h^{4}\left(\frac{116}{315} t^{7}+\frac{5}{315} t^{9}-\frac{43}{315} t^{8}+\frac{1259}{24192} t^{2}+\frac{16}{45} t^{5}-\frac{29}{5760} t-\frac{7579}{60480} t^{3}-\frac{8}{15} t^{6}-\frac{2}{945} t^{10}\right)
\end{aligned}
$$




$$
\begin{aligned}
& \beta_{2}(t)=h^{4}\left(-\frac{45107}{181440} t^{7}-\frac{1198717}{34836480} t^{2}-\frac{499}{2160} t^{5}-\frac{3055}{163296} t^{9}+\frac{11423}{120960} t^{8}+\frac{13693}{38880} t^{6}+\frac{83}{54432} t^{10}+\frac{41893}{11612160} t+\frac{4231973}{52254720} t^{3}\right) \\
& \tau_{2}(t)=h^{5}\left(-\frac{1}{1296} t^{9}+\frac{5}{1344} t^{8}+\frac{1}{15120} t^{10}+\frac{13}{92160} t+\frac{1681}{580608} t^{3}+\frac{7}{540} t^{6}-\frac{2431}{1935360} t^{2}-\frac{1}{120} t^{5}-\frac{19}{2016} t^{7}\right) \\
& \gamma_{2}(t)=h^{6}\left(\frac{517}{6048} t^{7}+\frac{181}{27216} t^{9}-\frac{19}{576} t^{8}-\frac{5}{9072} t^{10}-\frac{349}{276480} t-\frac{47323}{1741824} t^{3}-\frac{155}{1296} t^{6}+\frac{13535}{1161216} t^{2}+\frac{7}{90} t^{5}\right) .
\end{aligned}
$$

Evaluating the continuous method at the endpoint i.e. at $x_{n+2}$ yields equation (11)

$$
y_{n+2}=4 y_{n+\frac{3}{2}}+4 y_{n+\frac{1}{2}}-6 y_{n+1}-y_{n}+\frac{h^{4}}{5806080}\left[\begin{array}{l}
9871 f_{n+2}+48096 f_{n+\frac{3}{2}}+244296 f_{n+1}+ \\
60896 f_{n+\frac{1}{2}}-279 f_{n}-3750 h g_{n+2}+450 h^{2} m_{n+2}
\end{array}\right] \text {. }
$$

Evaluating the first, second and third derivatives of the continuous scheme at all the points give:

$$
\begin{aligned}
& y_{n}^{\prime}=\frac{1}{11612160 h}\left(\begin{array}{l}
1638 h^{6} m_{n+2}-14658 h^{5} g_{n+2}-12573 h^{4} f_{n}-6458 h^{4} f_{n+1}+41893 h^{4} f_{n+2}-269152 h^{4} f_{n+\frac{1}{2}} \\
-58464 h^{4} f_{n+\frac{3}{2}}-42577920 y_{n}-34836480 y_{n+1}+69672960 y_{n+\frac{1}{2}}+7741440 y_{n+\frac{3}{2}}
\end{array}\right) \\
& y_{n+\frac{1}{2}}^{\prime}=\frac{1}{69672960 h}\left(\begin{array}{l}
4086 h^{6} m_{n+2}-41178 h^{5} g_{n+2}+1143 h^{4} f_{n}+434808 h^{4} f_{n+1}+129809 h^{4} f_{n+2}+385216 h^{4} f_{n+\frac{1}{2}} \\
-225216 h^{4} f_{n+\frac{3}{2}}-46448640 y_{n}+139345920 y_{n+1}-69672960 y_{n+\frac{1}{2}}-23224320 y_{n+\frac{3}{2}}
\end{array}\right) \\
& y_{n+1}^{\prime}=-\frac{1}{34836480 h}\left(\begin{array}{l}
2358 h^{6} m_{n+2}-23466 h^{5} g_{n+2}-873 h^{4} f_{n}+290088 h^{4} f_{n+1}+7321 h^{4} f_{n+2}+123424 f_{n+\frac{1}{2}} \\
-122976 h^{4} f_{n+\frac{3}{2}}-11612160 y_{n}-34836480 y_{n+1}+69672960 y_{n+\frac{1}{2}}-23224320 y_{n+\frac{3}{2}}
\end{array}\right) \\
& y_{n+\frac{3}{2}}^{\prime}=\frac{1}{7741440 h}\left(\begin{array}{l}
738 h^{6} m_{n+2}-6942 h^{5} g_{n+2}-339 h^{4} f_{n}+187848 h^{4} f_{n+1}+20363 h^{4} f_{n+2}+54976 h^{4} f_{n+\frac{1}{2}} \\
-20928 h^{4} f_{n+\frac{3}{2}}-5160960 y_{n}-46448640 y_{n+1}+23224320 y_{n+\frac{1}{2}}+28385280 y_{n+\frac{3}{2}}
\end{array}\right) \\
& y_{n+2}^{\prime}=\frac{1}{34836480 h}\left(\begin{array}{l}
8010 h^{6} m_{n+2}-69774 h^{5} g_{n+2}-5355 h^{4} f_{n}+5640840 h^{4} f_{n+1}+227875 h^{4} f_{n+2}+1332320 h^{4} f_{n+\frac{1}{2}} \\
1876320 h^{4} f_{n+\frac{3}{2}}-127733760 y_{n}-661893120 y_{n+1}+487710720 y_{n+\frac{1}{2}}+301916160 y_{n+\frac{3}{2}}
\end{array}\right) \\
& y_{n}^{\prime \prime}=-\frac{1}{17418240 h^{2}}\left(\begin{array}{l}
43758 h^{6} m_{n+2}-406050 h^{5} g_{n+2}-278757 h^{4} f_{n}+200232 h^{4} f_{n+1} \\
1198717 h^{4} f_{n+2}-3298912 h^{4} f_{n+\frac{1}{2}}-1812960 h^{4} f_{n+\frac{3}{2}}-139345920 y_{n} \\
-278691840 y_{n+1}+348364800 y_{n+\frac{1}{2}}+69672960 y_{n+\frac{3}{2}}
\end{array}\right)
\end{aligned}
$$




$$
\begin{aligned}
& y_{n+\frac{1}{2}}^{\prime \prime}=\frac{1}{34836480 h^{2}}\left(\begin{array}{l}
4194 h^{6} m_{n+2}-38910 h^{5} g_{n+2}-18531 h^{4} f_{n}+59400 h^{4} f_{n+1}+ \\
114779 h^{4} f_{n+2}-709184 h^{4} f_{n+\frac{1}{2}}-172224 h^{4} f_{n+\frac{3}{2}}+139345920 y_{n} \\
+139345920 y_{n+1}-278691840 y_{n+\frac{1}{2}}
\end{array}\right) \\
& y_{n+1}^{\prime \prime}=-\frac{1}{5806080 h^{2}}\left(\begin{array}{l}
126 h^{6} m_{n+2}-1050 h^{5} g_{n+2}-201 h^{4} f_{n}+112824 h^{4} f_{n+1}+2641 h^{4} f_{n+2}+ \\
4640 h^{4} f_{n+\frac{1}{2}}+1056 h^{4} f_{n+\frac{3}{2}}+46448640 y_{n+1}-23224320 y_{n+\frac{1}{2}}-23224320 y_{n+\frac{3}{2}}
\end{array}\right) \\
& y_{n+\frac{3}{2}}^{\prime \prime}=\frac{1}{34836480 h^{2}}\left(\begin{array}{l}
11034 h^{6} m_{n+2}-87990 h^{5} g_{n+2}-6975 h^{4} f_{n}+5827464 h^{4} f_{n+1}+203191 h^{4} f_{n+2}+1464896 h^{4} f_{n+\frac{1}{2}} \\
494784 h^{4} f_{n+\frac{3}{2}}-139345920 y_{n}-696729600 y_{n+1}+557383680 y_{n+\frac{1}{2}}+278691840 y_{n+\frac{3}{2}}
\end{array}\right) \\
& y_{n+2}^{\prime \prime}=\frac{1}{17418240 h^{2}}\left(\begin{array}{l}
8442 h^{6} m_{n+2}-111750 h^{5} g_{n+2}-4527 h^{4} f_{n}+6656472 h^{4} f_{n+1}+676375 h^{4} f_{n+2}+1440992 h^{4} f_{n+\frac{1}{2}} \\
+3931488 h^{4} f_{n+\frac{3}{2}}-139345920 y_{n}-557383680 y_{n+1}+487710720 y_{n+\frac{1}{2}}+209018880 y_{n+\frac{3}{2}}
\end{array}\right) \\
& y_{n}^{\prime \prime \prime}=\frac{1}{8709120 h^{3}}\left(\begin{array}{l}
151290 h^{6} m_{n+2}-1419690 h^{5} g_{n+2}-1287333 h^{4} f_{n}+3041280 h^{4} f_{n+1}+4231973 h^{4} f_{n+2} \\
-5969504 h^{4} f_{n+\frac{1}{2}}-6548256 h^{4} f_{n+\frac{3}{2}}-69672960 y_{n}-209018880 y_{n+1}+209018880 y_{n+\frac{1}{2}} \\
69672960 y_{n+\frac{3}{2}}
\end{array}\right) \\
& y_{n+\frac{1}{2}}^{\prime \prime \prime}=-\frac{1}{8709120 h^{3}}\left(\begin{array}{l}
30690 h^{6} m_{n+2}-300930 h^{5} g_{n+2}-37665 h^{4} f_{n}+1963872 h^{4} f_{n+1}+929089 h^{4} f_{n+2} \\
+871136 h^{4} f_{n+\frac{1}{2}}-1549152 h^{4} f_{n+\frac{3}{2}}+69672960 y_{n}+209018880 y_{n+1} \\
-209018880 y_{n+\frac{1}{2}}-69672960 y_{n+\frac{3}{2}}
\end{array}\right) \\
& y_{n+1}^{\prime \prime \prime}=\frac{1}{8709120 h^{3}}\left(\begin{array}{l}
21690 h^{6} m_{n+2}-218730 h^{5} g_{n+2}-9477 h^{4} f_{n}+1921536 h^{4} f_{n+1}+691589 h^{4} f_{n+2} \\
+813472 h^{4} f_{n+\frac{1}{2}}-1239840 h^{4} f_{n+\frac{3}{2}}-69672960 y_{n}-209018880 y_{n+1}+ \\
209018880 y_{n+\frac{1}{2}}+69672960 y_{n+\frac{3}{2}}
\end{array}\right) \\
& y_{n+\frac{3}{2}}^{\prime \prime \prime}=-\frac{1}{8709120 h^{3}}\left(\begin{array}{l}
9090 h^{6} m_{n+2}-120930 h^{5} g_{n+2}-81 h^{4} f_{n}-3811104 h^{4} f_{n+1}+ \\
479473 h^{4} f_{n+2}-697120 h^{4} f_{n+\frac{1}{2}}-2503008 h^{4} f_{n+\frac{3}{2}}+69672960 y_{n} \\
+209018880 y_{n+1}-209018880 y_{n+\frac{1}{2}}-69672960 y_{n+\frac{3}{2}}
\end{array}\right)
\end{aligned}
$$




$$
y_{n+2}^{\prime \prime \prime}=\frac{1}{8709120 h^{3}}\left(\begin{array}{l}
13050 h^{6} m_{n+2}-267690 h^{4} g_{n+2}-1701 h^{4} f_{n}+3704832 h^{4} f_{n+1}+ \\
2399525 h^{4} f_{n+2}+715168 h^{4} f_{n+\frac{1}{2}}+4068576 h^{4} f_{n+\frac{3}{2}}-69672960 y_{n} \\
-209018880 y_{n+1}+209018880 y_{n+\frac{1}{2}}+69672960 y_{n+\frac{3}{2}}
\end{array}\right) .
$$

These schemes were combined in matrix form through matrix inversion; and a block method was produced in the following form:

$$
\begin{aligned}
& {\left[\begin{array}{llll}
1 & 0 & 0 & 0 \\
0 & 1 & 0 & 0 \\
0 & 0 & 1 & 0 \\
0 & 0 & 0 & 1
\end{array}\right]\left[\begin{array}{c}
y_{n+\frac{1}{2}} \\
y_{n+1} \\
y_{n+\frac{3}{2}} \\
y_{n+2}
\end{array}\right]=\left[\begin{array}{llll}
0 & 0 & 0 & 1 \\
0 & 0 & 0 & 1 \\
0 & 0 & 0 & 1 \\
0 & 0 & 0 & 1
\end{array}\right]\left[\begin{array}{c}
y_{n-\frac{1}{2}} \\
y_{n-1} \\
y_{n-\frac{3}{2}} \\
y_{n}
\end{array}\right]+h\left[\begin{array}{llll}
0 & 0 & 0 & \frac{1}{2} \\
0 & 0 & 0 & 1 \\
0 & 0 & 0 & \frac{3}{2} \\
0 & 0 & 0 & 2
\end{array}\right]\left[\begin{array}{c}
y^{\prime} \\
n-\frac{1}{2} \\
y_{n-1}^{\prime} \\
y^{\prime} \\
n-\frac{3}{2} \\
y_{n-2}^{\prime}
\end{array}\right]+h^{2}\left[\begin{array}{llll}
0 & 0 & 0 & \frac{1}{8} \\
0 & 0 & 0 & \frac{1}{2} \\
0 & 0 & 0 & \frac{9}{8} \\
0 & 0 & 0 & 2
\end{array}\right]\left[\begin{array}{c}
y_{n-\frac{1}{2}}^{\prime \prime} \\
y_{n-1}^{\prime \prime} \\
y^{\prime \prime} \\
y_{n-\frac{3}{2}}^{\prime \prime}
\end{array}\right]+} \\
& \left.h^{3}\left[\begin{array}{cccc}
0 & 0 & 0 & \frac{1}{48} \\
0 & 0 & 0 & \frac{1}{6} \\
0 & 0 & 0 & \frac{9}{16} \\
0 & 0 & 0 & \frac{4}{3}
\end{array}\right]\left[\begin{array}{c}
y^{\prime \prime \prime} \\
n-\frac{1}{2} \\
y_{n-1}^{\prime \prime} \\
y^{\prime \prime \prime} \\
n-\frac{3}{2} \\
y_{n-2}^{\prime \prime \prime}
\end{array}\right]+h^{4}\left[\begin{array}{cccc}
0 & 0 & 0 & \frac{7}{4320} \\
0 & 0 & 0 & \frac{2143}{120960} \\
0 & 0 & 0 & \frac{19143}{286720} \\
0 & 0 & 0 & \frac{158}{945}
\end{array}\right]\left[\begin{array}{l}
f_{n-\frac{1}{2}} \\
f_{n-1} \\
f_{n-\frac{3}{2}} \\
f_{n}
\end{array}\right]+h^{4}\left[\begin{array}{cccc}
\frac{8}{3645} & -\frac{5917}{1935360} & \frac{139}{26880} & -\frac{173737}{52254720} \\
\frac{218}{5103} & -\frac{709}{15120} & \frac{74}{945} & -\frac{163901}{3265920} \\
\frac{1857}{8960} & -\frac{2511}{14336} & \frac{351}{1120} & -\frac{57723}{286720} \\
\frac{15104}{25515} & -\frac{368}{945} & \frac{256}{315} & -\frac{376}{729}
\end{array}\right]\left[\begin{array}{c}
f_{n+\frac{1}{2}} \\
f_{n+1} \\
f_{n+\frac{3}{2}} \\
f_{n+2}
\end{array}\right]\right\} \\
& +h^{5}\left[\begin{array}{cccc}
0 & 0 & 0 & \frac{19391}{17418240} \\
0 & 0 & 0 & \frac{9131}{544320} \\
0 & 0 & 0 & \frac{9657}{143360} \\
0 & 0 & 0 & \frac{1468}{8505}
\end{array}\right]\left[\begin{array}{c}
g_{n+\frac{1}{2}} \\
g_{n+1} \\
g_{n+\frac{3}{2}} \\
g_{n+2}
\end{array}\right]+h^{6}\left[\begin{array}{cccc}
0 & 0 & 0 & -\frac{275}{2322432} \\
0 & 0 & 0 & -\frac{323}{181440} \\
0 & 0 & 0 & -\frac{513}{71680} \\
0 & 0 & 0 & -\frac{52}{2835}
\end{array}\right]\left[\begin{array}{c}
m_{n+\frac{1}{2}} \\
m_{n+1} \\
m_{n+\frac{3}{2}} \\
m_{n+2}
\end{array}\right]
\end{aligned}
$$

By writing out the block in Equation (27) explicitly, we have

$$
\begin{gathered}
y_{n+\frac{1}{2}}=y_{n}+\frac{1}{2} h y_{n}^{\prime}+\frac{1}{8} h^{2} y_{n}^{\prime \prime}+\frac{1}{48} h^{3} y_{n}^{\prime \prime \prime}+h^{4}\left[\begin{array}{l}
\frac{7}{4320} f_{n}+\frac{8}{36452} f_{n+\frac{1}{2}}-\frac{5917}{1935360} f_{n+1}+\frac{139}{26880} f_{n+\frac{3}{2}} \\
-\frac{173737}{52254720} f_{n+2}+\frac{19391}{17418240} h g_{n+2}-\frac{275}{2322432} h^{2} m_{n+2}
\end{array}\right] \\
y_{n+1}=y_{n}+y_{n}^{\prime} h+\frac{1}{2} h^{2} y_{n}^{\prime \prime}+\frac{1}{6} h^{3} y_{n}^{\prime \prime \prime}+h^{4}\left[\begin{array}{l}
\frac{2143}{120960} f_{n}+\frac{218}{5103} f_{n+\frac{1}{2}}-\frac{709}{15120} f_{n+1}+\frac{74}{945} f_{n+\frac{3}{2}} \\
-\frac{163901}{3265920} f_{n+2}+\frac{9131}{544320} h g_{n+2}-\frac{323}{181440} h^{2} m_{n+2}
\end{array}\right]
\end{gathered}
$$




$$
\begin{gathered}
y_{n+\frac{3}{2}}=y_{n}+\frac{3}{2} h y_{n}^{\prime}+\frac{9}{8} h^{2} y_{n}^{\prime \prime}+\frac{9}{16} h^{3} y_{n}^{\prime \prime \prime}+h^{4}\left[\begin{array}{l}
\frac{19143}{286720} f_{n}+\frac{1857}{8960} f_{n+\frac{1}{2}}-\frac{2511}{14336} f_{n+1}+\frac{351}{1120} f_{n+\frac{3}{2}} \\
-\frac{57723}{286720} f_{n+2}+\frac{9657}{143360} h g_{n+2}-\frac{513}{71680} h^{2} m_{n+2}
\end{array}\right] \\
y_{n+2}=y_{n}+2 h y_{n}^{\prime}+2 h^{2} y_{n}^{\prime \prime}+\frac{4}{3} h^{3} y_{n}^{\prime \prime \prime}+h^{4}\left[\begin{array}{l}
\frac{158}{945} f_{n}+\frac{15104}{25515} f_{n+\frac{1}{2}}-\frac{368}{945} f_{n+1}+\frac{256}{315} f_{n+\frac{3}{2}} \\
-\frac{376}{729} f_{n+2}+\frac{1468}{8505} h g_{n+2}-\frac{52}{2835} h^{2} m_{n+2}
\end{array}\right]
\end{gathered}
$$

Substituting the above Equations (28) - (31) into Equations (12) - (26) yield Equations (32) - (43)

$$
\begin{aligned}
& y_{n+\frac{1}{2}}^{\prime}=y_{n}^{\prime}+\frac{1}{2} h y_{n}^{\prime \prime}+\frac{1}{8} h^{2} y_{n}^{\prime \prime \prime}+h^{3}\left[\begin{array}{l}
\frac{5}{432} f_{n}+\frac{42869}{2177280} f_{n+\frac{1}{2}}-\frac{8419}{322560} f_{n+1}+\frac{10583}{241920} f_{n+\frac{3}{2}} \\
-\frac{244511}{8709120} f_{n+2}+\frac{779}{82944} h g_{n+2}-\frac{1931}{1935360} h^{2} m_{n+2}
\end{array}\right] \\
& y_{n+1}^{\prime}=y_{n}^{\prime}+h y_{n}^{\prime \prime}+\frac{1}{2} h^{2} y_{n}^{\prime \prime \prime}+h^{3}\left[\begin{array}{l}
\frac{3569}{60480} f_{n}+\frac{1471}{8505} f_{n+\frac{1}{2}}-\frac{209}{1260} f_{n+1}+\frac{53}{189} f_{n+\frac{3}{2}} \\
-\frac{97897}{544320} f_{n+2}+\frac{1091}{18144} h g_{n+2}-\frac{193}{30240} h^{2} m_{n+2}
\end{array}\right] \\
& y_{n+\frac{3}{2}}^{\prime}=y_{n}^{\prime}+\frac{3}{2} h y_{n}^{\prime \prime}+\frac{9}{8} h^{2} y_{n}^{\prime \prime \prime}+h^{3}\left[\begin{array}{l}
\frac{20547}{143360} f_{n}+\frac{927}{1792} f_{n+\frac{1}{2}}-\frac{12393}{35840} f_{n+1}+\frac{6201}{8960} f_{n+\frac{3}{2}} \\
-\frac{63711}{143360} f_{n+2}+\frac{2133}{14336} h g_{n+2}-\frac{81}{5120} h^{2} m_{n+2}
\end{array}\right] \\
& y_{n+2}^{\prime}=y_{n}^{\prime}+2 h y_{n}^{\prime \prime}+2 h^{2} y_{n}^{\prime \prime \prime}+h^{3}\left[\begin{array}{l}
\frac{50}{189} f_{n}+\frac{256}{243} f_{n+\frac{1}{2}}-\frac{32}{63} f_{n+1}+\frac{256}{189} f_{n+\frac{3}{2}} \\
-\frac{202}{243} f_{n+2}+\frac{158}{567} h g_{n+2}-\frac{4}{135} h^{2} m_{n+2}
\end{array}\right] \\
& y_{n+\frac{1}{2}}^{\prime \prime}=y_{n}^{\prime \prime}+\frac{1}{2} h y_{n}^{\prime \prime \prime}+h^{2}\left[\begin{array}{l}
\frac{74023}{1290240} f_{n}+\frac{4825}{36288} f_{n+\frac{1}{2}}-\frac{8677}{53760} f_{n+1}+\frac{5381}{20160} f_{n+\frac{3}{2}} \\
-\frac{1983911}{11612160} f_{n+2}+\frac{22093}{387072} h g_{n+2}-\frac{781}{129024} h^{2} m_{n+2}
\end{array}\right] \\
& y_{n+1}^{\prime \prime}=y_{n}^{\prime \prime}+h y_{n}^{\prime \prime \prime}+h^{2}\left[\begin{array}{l}
\frac{443}{3360} f_{n}+\frac{52}{105} f_{n+\frac{1}{2}}-\frac{5}{14} f_{n+1}+\frac{68}{105} f_{n+\frac{3}{2}} \\
-\frac{1403}{3360} f_{n+2}+\frac{47}{336} h g_{n+2}-\frac{5}{336} h^{2} m_{n+2}
\end{array}\right] \\
& y_{n+\frac{3}{2}}^{\prime \prime}=y_{n}^{\prime \prime}+\frac{3}{2} h y_{n}^{\prime \prime \prime}+h^{2}\left[\begin{array}{l}
\frac{4209}{20480} f_{n}+\frac{1973}{2240} f_{n+\frac{1}{2}}-\frac{6183}{17920} f_{n+1}+\frac{465}{448} f_{n+\frac{3}{2}} \\
-\frac{93791}{143360} f_{n+2}+\frac{3135}{14336} h g_{n+2}-\frac{333}{14336} h^{2} m_{n+2}
\end{array}\right]
\end{aligned}
$$




$$
\begin{aligned}
& y_{n+2}^{\prime \prime}=y_{n}^{\prime \prime}+2 h y_{n}^{\prime \prime \prime}+h^{2}\left[\begin{array}{l}
\frac{88}{315} f_{n}+\frac{512}{405} f_{n+\frac{1}{2}}-\frac{32}{105} f_{n+1}+\frac{512}{315} f_{n+\frac{3}{2}} \\
-\frac{70}{81} f_{n+2}+\frac{8}{27} h g_{n+2}-\frac{2}{63} h^{2} m_{n+2}
\end{array}\right] \\
& y_{n+\frac{1}{2}}^{\prime \prime \prime}=y_{n}^{\prime \prime \prime}+h\left[\frac{8179}{53760} f_{n}+\frac{13277}{22680} f_{n+\frac{1}{2}}-\frac{1931}{3360} f_{n+1}+\frac{781}{840} f_{n+\frac{3}{2}}-\frac{860177}{1451520} f_{n+2}+\frac{9559}{48384} h g_{n+2}-\frac{337}{16128} h^{2} m_{n+2}\right] \\
& y_{n+1}^{\prime \prime \prime}=y_{n}^{\prime \prime \prime}+h\left[\frac{493}{3360} f_{n}+\frac{13277}{22680} f_{n+\frac{1}{2}}-\frac{9}{70} f_{n+1}+\frac{64}{105} f_{n+\frac{3}{2}}-\frac{12293}{30240} f_{n+2}+\frac{139}{1008} h g_{n+2}-\frac{5}{336} h^{2} m_{n+2}\right] \\
& y_{n+\frac{3}{2}}^{\prime \prime \prime}=y_{n}^{\prime \prime \prime}+h\left[\frac{2649}{17920} f_{n}+\frac{643}{840} f_{n+\frac{1}{2}}+\frac{99}{1120} f_{n+1}+\frac{291}{280} f_{n+\frac{3}{2}}-\frac{29083}{53760} f_{n+2}+\frac{317}{1792} h g_{n+2}-\frac{33}{1792} h^{2} m_{n+2}\right] \\
& y_{n+2}^{\prime \prime \prime}=y_{n}^{\prime \prime \prime}+h\left[\frac{31}{210} f_{n}+\frac{2176}{2835} f_{n+\frac{1}{2}}+\frac{8}{105} f_{n+1}+\frac{128}{105} f_{n+\frac{3}{2}}-\frac{1193}{5670} f_{n+2}+\frac{25}{189} h g_{n+2}-\frac{1}{63} h^{2} m_{n+2}\right] .
\end{aligned}
$$

\section{ANALYSIS OF THE PROPERTIES OF THE BLOCK}

\subsection{Order and Error Constant of the Block}

In agreement with authors [5] and [20], we described the local truncation error related to a linear multistep method to be the linear difference operator. That is,

$$
L[y(x) ; h]=\sum_{j=0}^{k}\left\{a_{j} y(x+j h)-h^{4} \beta_{j} y^{i v}(x+j h)\right\} .
$$

Assuming that $y(x)$ is sufficiently differentiable, we can expand the terms in (44) above as a Taylor series about $x$ to obtain the expression:

$$
L[y(x) ; h]=C_{0} y(x)+C_{1} h y^{\prime}+\ldots,+C_{q} h^{q} y^{q}(x)+\ldots,
$$

where the constant coefficients $C_{q}, q=0,1, \ldots$ are given as follows:

$$
\begin{aligned}
& C_{0}=\sum_{j=0}^{k} \alpha_{j}, \\
& C_{1}=\sum_{j=0}^{k} j \alpha_{j}, \\
& \vdots \\
& C_{q}=\frac{1}{q !}\left[\sum_{j=1}^{k} j^{q} \alpha_{j}-(q-4) ! \sum_{j=1}^{k} j^{q-4} \beta_{j}\right] .
\end{aligned}
$$

According to [18], we say that our block is of uniform order $p=7$ and error constants given by the vector $C_{11}=\left[\frac{113437}{858370867200}, \frac{653}{335301120}, \frac{27801}{3532390400}, \frac{53}{532619540}\right]^{T}$. 


\subsection{Zero Stability of the Block Method}

Assuming the general form of the block method:

$A^{(0)} Y_{m}=A^{(i)} Y_{m-1}+h^{\mu}\left[B^{(i)} F_{m}+B^{(0)} F_{m-1}\right]$.

A block method is assumed to be zero stable, if the roots:

$\operatorname{det}\left[\lambda A^{(0)}-A^{(i)}\right]=0$

of the first characteristic polynomial fulfil $|\lambda| \leq 1$, and for the roots with $|\lambda| \leq 1$, the multiplicity must not surpass the order of the differential equation.

For our block,

$$
A=\left[z\left(\begin{array}{llll}
1 & 0 & 0 & 0 \\
0 & 1 & 0 & 0 \\
0 & 0 & 1 & 0 \\
0 & 0 & 0 & 1
\end{array}\right)-\left(\begin{array}{llll}
0 & 0 & 0 & 1 \\
0 & 0 & 0 & 1 \\
0 & 0 & 0 & 1 \\
0 & 0 & 0 & 1
\end{array}\right)\right]=0
$$

$A=z^{4}-z^{4}=0, z=0,0,0,0$.

This means that the block is zero stable.

\subsection{Region of Absolute Stability}

The stability nature of the method is found in the spirit of [5] and [21] shown in Figure (1) below:

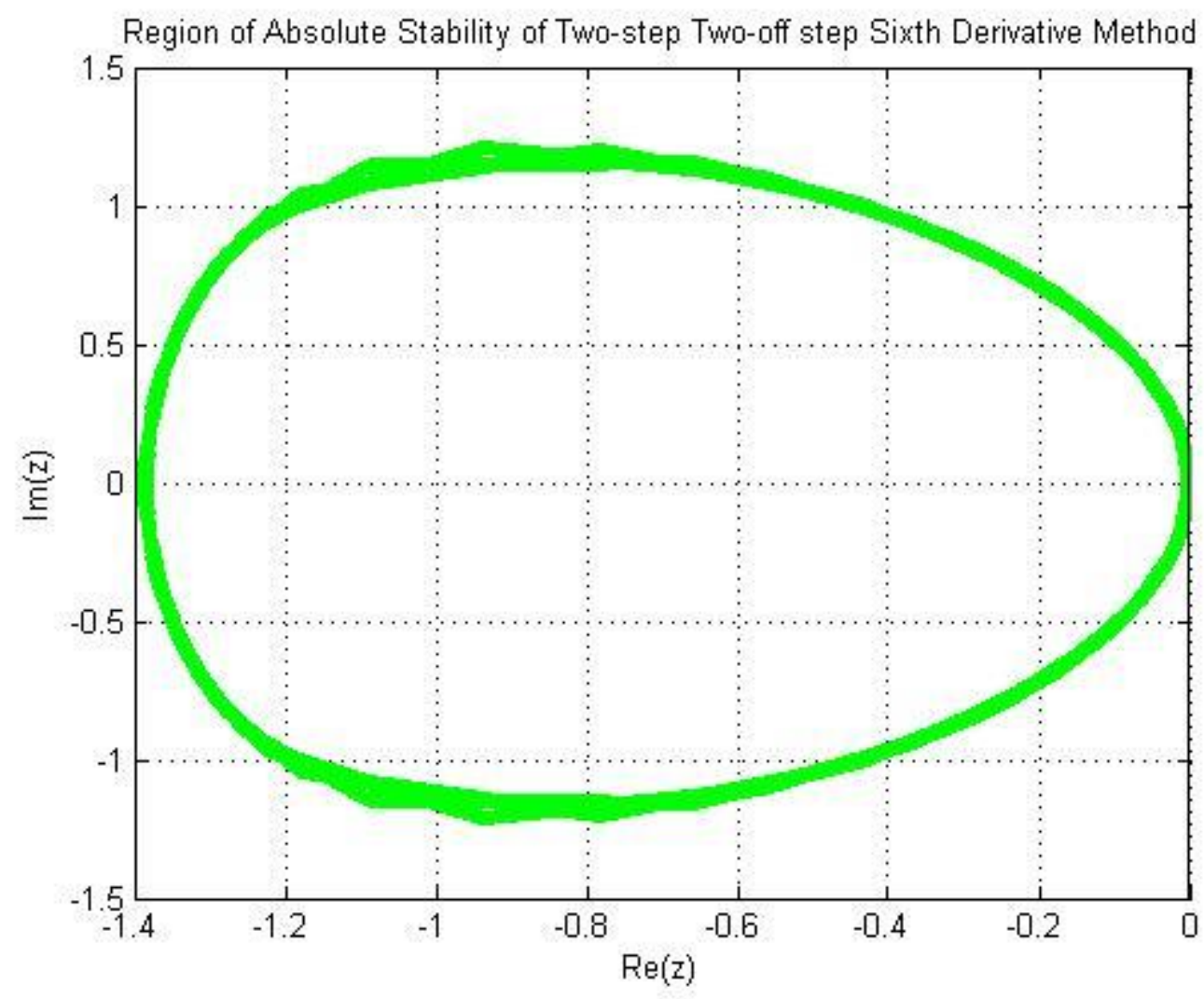

Figure 1. Showing the region of absolute stability of our method 


\subsection{Convergence}

Theorem 1: Convergence [5] - The necessary and sufficient condition for a linear multistep method to be convergent is for it to be consistent and zero stable. From the theorem above, the new hybrid block method is convergent.

\section{NUMERICAL RESULTS}

Here in this section, the performance of the new 2-step hybrid method was observed on some test examples. The results obtained from the test examples are shown in tabular form.

\section{Example 1.}

We considered the non-homogeneous example:

$y^{\prime \prime}=-100 y+99 \sin x, \quad y(0)=1, y^{\prime}(0)=11, \quad h=0.003125$

Exact solution: $y=\sin (10 x)+\cos (10 x)+\sin x$

Source: [22].

Table 1. Showing the comparison of the result for test example 1 with the error in method [22]

\begin{tabular}{|l|l|l|l|l|}
\hline$x$ & Exact Solution & New Scheme Solution & Error & $\begin{array}{l}\text { Error in Method } \\
{[22] k=1}\end{array}$ \\
\hline 0.003125 & 1.03388166738420191 & 1.03388166738426647 & $6.45600 \mathrm{E}-14$ & $7.9800 \mathrm{E}-11$ \\
\hline 0.006250 & 1.06675678785245466 & 1.06675678785236532 & $8.93400 \mathrm{E}-14$ & - \\
\hline 0.009375 & 1.09859628036501657 & 1.09859628036472113 & $2.95440 \mathrm{E}-13$ & $8.3780 \mathrm{E}-10$ \\
\hline 0.012500 & 1.12937207509626653 & 1.12937207509542852 & $8.38010 \mathrm{E}-13$ & - \\
\hline 0.015625 & 1.15905714081491135 & 1.15905714081343779 & $1.47356 \mathrm{E}-12$ & - \\
\hline 0.018750 & 1.18762551125002438 & 1.18762551124751685 & $2.50753 \mathrm{E}-12$ & $3.3600 \mathrm{E}-09$ \\
\hline 0.021875 & 1.21505231041716944 & 1.21505231041349118 & $1.54455 \mathrm{E}-12$ & - \\
\hline 0.025000 & 1.21431377687988004 & 1.21431377687456903 & $5.31101 \mathrm{E}-12$ & - \\
\hline 0.028125 & 1.26638728692280306 & 1.26638728691567611 & $7.12695 \mathrm{E}-12$ & $7.3481 \mathrm{E}-09$ \\
\hline 0.031250 & 1.29025137661387913 & 1.29025137660440947 & $9.46966 \mathrm{E}-12$ & - \\
\hline
\end{tabular}

\section{Example 2.}

$$
y^{\prime \prime \prime}=-e^{x}, \quad y(0)=1, y^{\prime}(0)=-1, y^{\prime \prime}(0)=3, h=0.1
$$

Exact Solution: $y(x)=2+2 x^{2}-e^{-x}$

Source: [23].

Table 2. Showing the comparison of the result for test example 2 with the error in method [23]

\begin{tabular}{|l|l|l|l|l|}
\hline$x$ & Exact Solution & New Scheme Solution & Error & $\begin{array}{l}\text { Error in Method } \\
{[23] k=4}\end{array}$ \\
\hline 0.1 & 0.91482908192435238 & 0.914829081924317644 & $3.473600 \mathrm{E}-14$ & $2.5080 \mathrm{E}-13$ \\
\hline 0.2 & 0.85859724183983017 & 0.858597241840162860 & $3.326900 \mathrm{E}-13$ & $6.4932 \mathrm{E}-11$ \\
\hline 0.3 & 0.83014119242399690 & 0.830141192420339910 & $3.709100 \mathrm{E}-14$ & $1.6831 \mathrm{E}-09$ \\
\hline 0.4 & 0.82817530235872968 & 0.828175302359308864 & $5.791840 \mathrm{E}-13$ & $3.3668 \mathrm{E}-09$ \\
\hline 0.5 & 0.85127872929987185 & 0.851278729300229951 & $3.581010 \mathrm{E}-13$ & $6.6147 \mathrm{E}-09$ \\
\hline
\end{tabular}




\begin{tabular}{|l|l|l|l|l|}
\hline 0.6 & 0.89788119960949103 & 0.897881199610700328 & $1.209298 \mathrm{E}-12$ & $9.9982 \mathrm{E}-09$ \\
\hline 0.7 & 0.96624729252952348 & 0.966247292530703475 & $1.179995 \mathrm{E}-12$ & $1.5283 \mathrm{E}-08$ \\
\hline 0.8 & 1.05445907150753240 & 1.054459071510046900 & $2.514500 \mathrm{E}-12$ & $2.1980 \mathrm{E}-08$ \\
\hline 0.9 & 1.16039688884305034 & 1.160396888845880500 & $2.409110 \mathrm{E}-12$ & $3.4643 \mathrm{E}-08$ \\
\hline 1.0 & 1.28171817154095476 & 1.281718171545825430 & $4.870670 \mathrm{E}-12$ & $5.9998 \mathrm{E}-08$ \\
\hline
\end{tabular}

\section{Example 3.}

We considered the special fourth order problem

$$
y^{i v}+y^{\prime \prime}=0, y(0)=0, y^{\prime}(0)=\left(-\frac{1.1}{72-50 \pi}\right), y^{\prime \prime}(0)=\left(\frac{1}{144-100 \pi}\right), y^{\prime \prime \prime}(0)=\left(-\frac{1.2}{144-100 \pi}\right), h=\frac{0.1}{32}
$$

Exact Solution: $y(x)=\frac{1-x-\cos x-1.2 \sin x}{144-100 \pi}$

Source: [24].

Table 3. Showing the comparison of the result for test example 3 with the error in method [24]

\begin{tabular}{|l|l|l|l|l|}
\hline$x$ & Exact Solution & New Scheme Solution & Error & $\begin{array}{l}\text { Error in Method } \\
\text { [24] } k=1\end{array}$ \\
\hline 0.103150 & 0.0000403745930229973261 & 0.0000403745930229994393 & $2.11320 \mathrm{E}-18$ & $0.38142683 \mathrm{E}-18$ \\
\hline 0.206250 & 0.0000806915800710702613 & 0.0000806915800710702613 & $1.05766 \mathrm{E}-17$ & $0.37184370 \mathrm{E}-17$ \\
\hline 0.306250 & 0.000120950746770959792 & 0.000120950746771004095 & $4.43030 \mathrm{E}-17$ & $0.26822346 \mathrm{E}-16$ \\
\hline 0.406250 & 0.000161151879314058272 & 0.000161151879314125709 & $6.74370 \mathrm{E}-17$ & $0.29384802 \mathrm{E}-16$ \\
\hline 0.506250 & 0.000201294764458497415 & 0.000201294764458612830 & $1.15415 \mathrm{E}-16$ & $0.41813224 \mathrm{E}-15$ \\
\hline 0.603125 & 0.000241379189531230713 & 0.000241379189531382827 & $1.52114 \mathrm{E}-16$ & $0.38734880 \mathrm{E}-15$ \\
\hline 0.703125 & 0.000281404942430110347 & 0.000281404942430323570 & $2.13223 \mathrm{E}-16$ & $0.28714827 \mathrm{E}-15$ \\
\hline 0.803125 & 0.000321371811625958463 & 0.000321371811626220817 & $2.62354 \mathrm{E}-16$ & $0.86740034 \mathrm{E}-14$ \\
\hline 0.903125 & 0.000361279586164632919 & 0.000361279586164968374 & $3.35455 \mathrm{E}-16$ & $0.70802448 \mathrm{E}-14$ \\
\hline 1.003125 & 0.000401128055669087341 & 0.000401128055669483216 & $3.95875 \mathrm{E}-16$ & $0.35121472 \mathrm{E}-14$ \\
\hline
\end{tabular}

\section{DISCUSSION OF RESULTS AND CONCLUSION}

In table 1, our new scheme was applied on a non-homogenous second order initial value problem (IVP) ODE which had been solved by [22]. Our new method generated more correct results compared to the Onestep hybrid block method executed by [22]. The solution of a third order IVP ODE executed by our new hybrid method is shown in table 2 and it is evident that our method performs better than that of [23]'s fourstep scheme. Table 3 shows the comparison of the result of our method with [24]'s One-step hybrid block method on a special fourth order IVP ODE. However, our scheme is found to be more accurate than [24]'s.

In conclusion, we propose a two-step scheme with two hybrid points for the direct solution of second, third and fourth order IVPs of ordinary differential equations. From the three test examples solved by the new scheme; it has been established that it is effective in handling second, third and fourth order ordinary differential equations initial value problems directly. This finding is seen from the accuracy of the numerical results presented so far. Hence it is efficient, accurate and reliable. 


\section{ACKNOWLEDGEMENTS}

The authors wish to appreciate the editor and anonymous referees for their valuable comments and suggestions which have further improved the quality of this work.

\section{CONFLICTS OF INTEREST}

No conflict of interest was declared by the authors.

\section{REFERENCES}

[1] Waeleh, N., Majid, Z. A., Ismail, F. and Suleiman, M., "Numerical solution of higher order ordinary differential equations by direct block code", Journal of Mathematics and Statistics, 8 (1): 77-81, (2012).

[2] Cakır, M. and Arslan, D., "Reduced differential transform method for singularly perturbed sixthorder boussinesq equation”, Math Stat ,2: 14, 1-8, (2016).

[3] Arslan, D., "A novel hybrid method for singularly perturbed delay differential equations", Gazi University Journal of Science, 32(1): 217 - 223, (2019).

[4] Cakir, M., Masiha, Y. R. and Arslan, D., "A novel numerical approach for solving convectiondiffusion problem with boundary layer behavior", Gazi University Journal of Science, 33 (1): 152 $-166,(2020)$.

[5] Lambert, J. D., "Computational Methods in Ordinary Differential Equation", John Wiley \& Sons Inc., New York, (1973).

[6] Awoyemi, D. O., "A class of continuous linear multistep methods for general second order initial value problems in ordinary differential equations", International Journal of Computer Mathematics, 72: 29-37, (1999).

[7] Brugnano, L. and Trigiante, D., "Solving Differential Problems by Multistep IVPs and BVP Methods", Gordon and Breach Science Publishers, (1998).

[8] Gear, C. W., "The numerical integration of ordinary differential equations", Math. Comp., 21: 146 - 156, (1966).

[9] Gear, C. W., "Numerical Initial Value Problems in Ordinary Differential Equations”, New Jersey, Prentice Hall, (1971).

[10] Gear, C. W., "The stability of numerical methods for second order ordinary differential equations", SIAM Journal of Numerical Analysis, 15(1): 1187 - 197, (1987).

[11] Hall, G. and Suleiman, M. B., "Stability of Adams - type formulae for second order ordinary differential equations", SIAM Journal of Numerical Analysis, 1: 427 -428, (1981).

[12] Mohammed, U., "A six step block method for solution of fourth order ordinary differential equations", The Pacific Journal of Science and Technology, 11(1): 259-265, (2010).

[13] Badmus, A. M. and Yahaya, Y. A., "A class of collocation methods for general second order ordinary differential equations", African Journal of Mathematics and Computer Science Research, 2(4): 69-71, (2009).

[14] Ogunware, B. G., Omole, E. O. and Olanegan, O. O., "Hybrid and non-hybrid implicit schemes for solving third order odes using block method as predictors", Journal of Mathematical Theory and Modeling (IISTE), 5(3): 10-25, (2015). 
[15] Omole, E. O. and Ogunware, B. G., "3- point single hybrid block method (3pshbm) for direct solution of general second order initial value problem of ordinary differential equations", JSRR, 20(3): 1-11, (2018).

[16] Areo, E. A. and Omole, E. O., "Half-step symmetric continuous hybrid block method for the numerical solutions of fourth order ordinary differential equations", Archives of Applied Science Research, 7 (10): 39-49, (2015).

[17] Kuboye, J. O, Omar, Z., Abolarin, O. E. and Abdelrahim, R., "Generalized hybrid block method for solving second order ordinary differential equations directly", Res. Rep. Math. , 2(1): 1-7, (2018).

[18] Omar, Z. B. and Suleiman, M. B., "Solving higher order odes directly using parallel 2-point explicit block method", Matematika Pengintegrasian Teknologi Dalam Sains Matematik, Universiti Sains Malaysia, 21(1): 15-23, (2005).

[19] Majid, Z. A., "Parallel Block Methods for Solving Ordinary Differential Equations", PhD Thesis, University Putra Malaysia, 25-38 (2004).

[20] Fatunla, S.O., "Block methods for second order ivps”, Intern. J. Computer Math., 41(9): 55-63, (1991).

[21] Awoyemi, D. O., "A p-stable linear multistep method for solving third order ordinary differential equation”, Int. J. Compt, Math., 80(8): 85-99, (2003).

[22] Adeniran, A. O., Odejide, S. A. and Ogundare, S. B., "One step hybrid numerical scheme for the direct solution of general second order ordinary differential equations", International Journal of Applied Mathematics", 28(3): 197-212, (2015).

[23] Adoghe, L. O., Ogunware, B. G. and Omole, E. O., "A family of symmetric implicit higher order methods for the solution of third order initial value problems in ordinary differential equations", Theoretical Mathematics \& Applications, 6(3): 67-84, (2016).

[24] Duromola, M.K., "An accurate five off-step points 1mplicit block method for direct solution of fourth order differential equations", Open Access Library Journal, 3: 1-14, (2016). 